\title{
1 Umgang mit begrenzten Mitteln in deutschen Kliniken: empirische Befunde
}

\author{
Daniel Strech und Georg Marckmann
}

\subsection{Einführung}

Im Grunde bedürfte es keiner Empirie, um per se den Bedarf an ärztlicher Rationierung festzustellen. Viele Personen würden es als unplausibel deklarieren, dass Gesundheitssysteme (weltweit) sämtliche potenziell nützlichen medizinischen Maßnahmen allen hierfür infrage kommenden Patienten zur Verfügung stellen bzw. bezahlen können (Marckmann 2007; Fojo u. Grady 2009; Truog 2009; Kent 2010). Nun finden sich im deutschen Recht bzw. in der deutschen Rechtsprechung aber Sätze, die diese unplausible Auffassung nahelegen (Huster et al. 2007; Katzenmeier 2010). Zudem - oder gerade deshalb - scheint diese unplausible Auffassung (oder: Wunschvorstellung?) auch bei der Öffentlichkeit, der Gesetzlichen Krankenversicherung, der Ärzteschaft (zumindest bis zum Jahre 2008, s.u.) und bei politischen Funktionären weit verbreitet. Damit der Problembereich „Umgang mit begrenzten Ressourcen“ als praktisch relevant auch von diesen Personengruppen anerkannt wird, sind empirische Informationen wichtig, die zeigen, dass durch den medizinischen Fortschritt und den demographischen Wandel mit hoher Wahrscheinlichkeit eine weiter zunehmende Begrenzung der Mittel in Gesundheitswesen (weltweit) zu erwarten ist (Fojo u. Grady 2009; Beske 2010). Neben empirischen Informationen zur Realität des Kostendrucks, dürften empirische Daten zur Realität von ärztlicher Rationierung noch deutlicher aufzeigen, dass man sich dieser Thematik (fach-)politisch nicht verschließen kann. 
In den letzten Jahren wird das Thema Rationierung zunehmend auch in der deutschen Ärzteschaft diskutiert. Die Zentrale Ethikkommission bei der Bundesärztekammer (ZEKO) plädierte in einem Positionspapier vom September 2007 für eine explizite Priorisierung im Gesundheitswesen (ZEKO 2007). Im Mai 2008 wies die Bundesärztekammer in ihrem Ulmer Papier öffentlichkeitswirksam darauf hin, dass Rationierung gegenwärtig stattfinde und durch Rationalisierungen allein nicht zu verhindern sei (BÄK 2008). Dies wurde in einem Positionspapier der Ärzteschaft bestätigt (Fuchs 2010). Eine im Frühjahr 2012 vom Vorstand der Bundesärztekammer eingesetzte Arbeitsgruppe „Priorisierung im Gesundheitswesen " unterstrich, dass eine ärztlich unterstütze Priorisierung notwendig und hilfreich sei (Raspe u. Schulze 2013). Noch relevanter als die standespolitischen Stellungnahmen wären repräsentative Umfragestudien zur Frage, ob sich Ärzte aufgrund der Mittelbegrenzung in ihrer Tätigkeit bereits heute mit Priorisierungen und Rationierungen konkret konfrontiert sehen.

Ist das Problem Mittelbegrenzung in der gesundheitlichen Versorgung als solches erst einmal anerkannt, werden quantitative und qualitative empirische Studien in einem weniger politischen, sondern nunmehr wissenschaftlichen und analysierenden Sinn relevant. Dann nämlich, wenn sie die konzeptionellen und praktischen Problembereiche im Umgang mit begrenzten Mitteln untersuchen. Auf einer entsprechend validen empirischen Basis lassen sich ethische wie aber auch gesundheitsökonomische und gesundheitspolitische Vorschläge für eine praxisorientierte Verbesserung der Situation erarbeiten.

Dieser Beitrag zielt primär auf die deskriptive Darstellung der Befunde aus internationalen und nationalen sozialempirischen Untersuchungen. Eine ausführliche Diskussion der verschiedenen Ergebnisse ist in diesem Beitrag nicht vorgesehen. Siehe hierfür u.a. die anderen Beiträge in diesem Sammelband wie auch (Strech et al. 2010; Strech u. Marckmann 2010).

\subsection{Stand der qualitativen Forschung zum Umgang mit Mittelbegrenzung}

Verschiedene internationale qualitative Studien haben sich mit den Auswirkungen von Mittelknappheit auf die ärztliche Tätigkeit beschäftigt. Ian Rees Jones et al. zum Beispiel fokussieren in Interviews mit englischen Ärzten die Arzt-Patienten-Beziehung und die Frage nach der Möglichkeit eines informed consent bei der Berücksichtigung ökonomischer Aspekte (Jones et al. 2004). In einer Studie mit norwegischen Ärzten konnten Benedicte Carlsen und Ole Frithjof Norheim die Verunsicherungen von Ärzten hinsichtlich ihres beruflichen Selbstverständnisses bei der Notwendigkeit von Leistungsbegrenzungen aufzeigen (Carlsen $u$. Norheim 2005). Weitere internationale qualitative Studien zu ähnlichen Fragestellungen wurden von (Ayres 1996; Stronks et al. 1997; Hurst et al. 2005; Reeleder et al. 2005) durchgeführt. Die internationale 
qualitative Forschung wurde in einer systematischen Übersichtsarbeit zusammenfassend dargestellt in (Strech et al. 2008).

In drei deutschen qualitativen Studien mit Klinikpersonal konnte gezeigt werden, dass sich die Mittelknappheit in der klinischen Tätigkeit bereits heute niederschlägt. In der Arbeit von Ellen Kuhlmann lag der Fokus auf der Kommunikation ökonomischer Aspekte in der Arzt-Patienten-Beziehung (Kuhlmann 1999). Carlo Schultheiss untersuchte, welche Versorgungsbereiche heute möglicherweise der Rationierung unterliegen und welche Form eine etwaige Rationierung dort annimmt (Schultheiss 2004). Die Studie von Petra Buhr und Sebastian Klinke beschäftigte sich mit den Folgen der Einführung des DRG-Systems in deutschen Kliniken (Buhr u. Klinke 2006). Alle Studien konnten zeigen, dass sich ökonomische Aspekte auf die Patientenversorgung und die Arbeitsbedingungen in der Klinik auf unterschiedliche Weise auswirken. Eine genaue Analyse der Begriffe Rationierung und Mittelknappheit und der jeweils zur Anwendung kommenden Priorisierungskriterien war jedoch nicht primäres Ziel dieser Arbeiten.

\subsection{Stand der quantitativen Forschung zum Umgang mit Mittelbegrenzung}

Weltweit wiesen einige wenige Umfragestudien unter Ärzten nach, dass Rationierungsentscheidungen durch einzelne Ärzte im ambulanten wie stationären Bereich bereits heute stattfinden (Hurst et al. 2006; Ward et al. 2008). In der von Samia Hurst et al. durchgeführten länderübergreifenden Umfrage unter Ärzten in Italien, Großbritannien, Norwegen und der Schweiz wurde die individuelle Häufigkeit von Rationierungsmaßnahmen untersucht (Hurst et al. 2006). Dort berichteten 56\% der Ärzte, dass sie Maßnahmen rationieren müssten, jedoch gaben nur etwa $15 \%$ an, dass dies täglich oder wöchentlich geschehe.

Deutlich zahlreicher als Umfragen zum direkten Ausmaß an Rationierung sind Umfragestudien, welche die Bereitschaft von Ärzten zur Rationierung untersuchten (Strech et al. 2009). In einer systematischen Übersichtsarbeit, welche 15 Studien zu dieser Fragestellung identifizieren konnte, wurde aufgezeigt, dass die Bereitschaft zur Rationierung über die Studien hinweg von $9 \%$ bis 94\% streute (Strech et al. 2009). Diese starke Streubreite scheint nicht unerheblich durch die Formulierung der jeweiligen Fragebogenitems zur Erfassung der Rationierungsbereitschaft beeinflusst zu sein. So wurde eine eher hohe Rationierungsbereitschaft ermittelt, wenn relativ unverbindliche Fragen gestellt wurden wie etwa: „Cost is an important consideration when making medication choices“ (88\% Zustimmung in [Reichert et al. 20oo]) oder „Good quality prescribing takes into account the value for money of the medicines prescribed and not just their appropriateness, effectiveness and safety of treatment for the patient" (79\% Zustimmung in [Baines et al. 1998]). Sobald aller- 
dings die möglichen praktischen Konsequenzen von Rationierung genannt wurden oder konkrete Handlungen angesprochen wurden, sank die Rationierungsbereitschaft. So fand zum Beispiel die Aussage „Physicians need to adopt more cost saving measures even if such measures sometimes reduce quality of care" nur 28\% Zustimmung (Bernat et al. 1997), 37\% der Befragten stimmten der Aussage zu „I should sometimes deny beneficial but costly services to certain patients because resources should go to other patients that need them more“ (Hurst et al. 2006).

Aus Deutschland liegen zwei quantitative, in ihrer Repräsentativität allerdings deutlich eingeschränkte Umfragestudien unter Ärzten vor (Kern et al. 1999; Boldt u. Schöllhorn 2008). In der Umfrage von Axel Kern et al. unter Lesern des Deutschen Ärzteblatts gaben 73\% der Befragten an, es finde heute schon eine Rationierung von Gesundheitsleistungen statt. In der Studie von Joachim Boldt und Thilo Schöllhorn antworteten 67\% der befragten Leiter von Intensivstationen, dass Rationierungen bereits stattfänden. Beide Studien fragten jeweils explizit nach der Häufigkeit von „Rationierung“, ohne den Begriff dabei inhaltlich zu spezifizieren.

\subsection{Tiefeninterviews mit leitenden Ärzten im Rahmen des BMBF Forschungsverbunds „Allokation“}

Wer mithilfe von Fragebögen bzw. geschlossenen Fragen nach dem Vorhandensein von Rationierung oder der Relevanz des Lebensalters als Priorisierungskriterium fragt, fragt nach sehr komplexen Begriffen und Konzepten (Pearlman u. Starks 2001; Christianson et al. 2005). Um die Antworten in deutschen Fragebogenstudien besser interpretieren zu können und um Fragestellungen zu formulieren, welche überhaupt erst hilfreiche quantitative Informationen generieren können, bedarf es deshalb vorausgehend einer offenen Beantwortung folgender qualitativ zu beantwortender Fragen: Was verstehen deutsche Ärzte unter Mittelknappheit? Wie erleben sie diese? Was meinen Ärzte, wenn sie sagen, dass bereits heute rationiert werden muss bzw. dass noch nicht rationiert werden muss? Was meinen Ärzte, wenn sie sagen, dass die Prognose oder das Lebensalter eines Patienten relevante Kriterien bei der Allokation knapper Ressourcen darstellen?

Wie bereits dargestellt, haben die drei oben genannten qualitativen Studien in Deutschland zum Thema Mittelbegrenzung nicht primär darauf gezielt, die Verwendung der Begriffe Rationierung und Mittelknappheit und der jeweils zur Anwendung kommenden Priorisierungskriterien genauer zu analysieren.

In einer eigenen 2007 abgeschlossenen qualitativen Studie im Rahmen des BMBF Forschungsverbunds „Allokation“ wurden Tiefeninterviews mit Chefärzten und leitenden Oberärzten aus der interventionellen Kardiologie und 
der Intensivmedizin durchgeführt (Huster et al. 2007; Strech et al. 2008; Strech et al. 2009). Neben einer Erfassung des ärztlichen Verständnisses von Begriffen wie Rationierung und Lebensalter als Zuteilungskriterium war es ein zentrales Ziel dieser Studie, relevante Einflussfaktoren im Umgang mit Kostendruck und die gegenwärtig von Klinikern erlebten Konsequenzen in ihrer Spannbreite zu erfassen. Zum einen können die Aussagen der befragten Ärzte $\mathrm{zu}$ erschwerenden Bedingungen, Einflussfaktoren und Konsequenzen neue ethische Problemfelder aufdecken. Zum anderen bedarf es einer genauen Kenntnis des Denkens und Handelns der Akteure in der Praxis, um angemessene Lösungskonzepte für die ethischen Probleme zu erarbeiten, welche z.B. in Sinne einer Ethikfolgenabschätzung - die möglichen Hindernisse bei ihrer Implementierung berücksichtigen. Die Angemessenheit und Qualität einer theoretisch gut begründeten ethischen Strategie - wie z.B. auf Transparenz und Gleichbehandlung zielende Kostensensible Leitlinien - kann nicht unabhängig von den gegenwärtigen Praxisbedingungen beurteilt werden. Erwartungsgemäß werden einige ethisch relevante Praxisbedingungen für eine Implementierung expliziter Strategien sprechen, andere Praxisbedingungen hingegen können mehr oder weniger starke Hindernisse für eine erfolgreiche Implementierung darstellen.

\subsubsection{Methodik}

Insgesamt wurden 13 halbstrukturierte, leitfadengestützte Tiefeninterviews mit insgesamt 15 Ärzten in leitender Position aus den Fachbereichen Intensivmedizin und interventioneller Kardiologie an insgesamt 5 verschiedenen Kliniken durchgeführt. Der Leitfaden bestand aus thematisch relevanten Stichpunkten, welche der Formulierung offener Fragen dienten, um die Interviewpartner zu Erzählungen anzuregen. Der Inhalt des Leitfadens wurde mit den anderen Mitarbeitern des Forschungsverbundes „Allokation“abgestimmt. Die Interviews dauerten jeweils zwischen 45 und 70 Minuten, wurden aufTonband aufgezeichnet und vollständig transkribiert. Wie im Bereich der qualitativen Forschung üblich, wurde das Sample der Interviewpartner nach zweckdienlichen Aspekten zusammengestellt (purposive sampling). Als maßgeblich erschien uns die Berücksichtigung der verschiedenen institutionellen Rahmenbedingungen wie der Versorgungsauftrag (Uniklinik, Haus der Maximalversorgung, Haus der Regelversorgung) und der Krankenhausträger (öffentlich, privat). $\mathrm{Zu}$ weiteren spezifischeren Charakteristika siehe (Strech et al. 2008).

Die Auswertung der Interviews erfolgte nach den ersten beiden Stufen der Methodik der „Grounded Theory“ (Strauss u. Corbin 1996). Zunächst wurden alle Transkripte vollständig gelesen. Die Auswertung der ersten 9 Interviews diente der Erstellung eines Kategoriengerüstes (offenes Kodieren, erster Schritt der „Grounded Theory“). Hierbei wurden Textpassagen zentralen und möglichst abstrahierenden Themen bzw. Hauptkategorien zugeordnet. In einer zweiten Durchsicht und Analyse der Transkripte wurden die Hauptkategorien durch 
spezifischere Subkategorien erster und zweiter Ordnung in ihren Dimensionen erweitert (axiales Kodieren, zweiter Schritt der „Grounded Theory“). Sämtliche Haupt- und Subkategorien sind somit als Resultate einer systematischen Textinterpretation zu verstehen, welche auf den Originalzitaten aus den Interviews beruht. Teile dieser Originalzitate werden zur Verdeutlichung und Überprüfbarkeit unseres Kategoriengerüstes im Folgenden präsentiert.

Die Auswertung der weiteren 4 Interviews nach der gleichen Methodik ergänzte in einzelnen Fällen die Sublategorien zweiter Ordnung, führte aber zu keiner weiteren Ergänzung der Hauptkategorien und der Subkategorien erster Ordnung. Wir können somit von einem Hinweis für eine bessere Objektivität bezüglich des Kategoriengerüstes ausgehen. Nach dem Ansatz der „Grounded Theory" gelten diese Kategorienebenen als theoretisch gesättigt (theoretical saturation).

\subsubsection{Ergebnisse}

Die Tabellen 1 und 2 bieten eine Übersicht zu den ermittelten Hauptkategorien und Subkategorien, die im Folgenden skizziert werden.

Die Kategorien aus den Tabellen 1 und 2 basieren in der Regel auf verschiedenen Zitaten. Aufgrund der Materialfülle ist eine vollständige Präsentation aller Textpassagen aus dem Originalmaterial hier nicht möglich. Die Darstellung beschränkt sich deshalb auf einzelne Textpassagen. Zur Wahrung der Anonymität der Interviewteilnehmer werden keine näheren Angaben zur Herkunft der Zitate gemacht. Die Auswahl beschränkt sich nicht auf die prägnantesten oder „stimmigsten“ Zitate, sondern bezieht bewusst auch widersprüchliche Zitate mit ein.

\section{Mittelknappheit \& Rationierung}

Die Berichte der Interviewteilnehmer zeigen, wie vielschichtig der phänomenale Gehalt der Begriffe Mittelknappheit und Rationierung (auch Leistungsbegrenzung) ist. Zunächst lassen sich die Aussagen der Ärzte so interpretieren, dass sich die Mittelknappheit in direkter oder indirekter Form manifestiert. Direkt: ,mit den Mitteln, die wir jetzt hier im Hause auch haben, sehe ich keine Möglichkeit auch im Geringsten nur irgendetwas einzusparen, wir machen nur Basismedizin“; indirekt: „Die Knappheit spüre ich nicht unbedingt. Es gibt natürlich jährliche Sitzungen, wo man sich dann rechtfertigen muss bezüglich der Ausgaben und Budgets, ob man drüber oder drunter liegt.“

Insbesondere die jüngeren Ärzte berichteten, dass durch den Umgang mit Mittelknappheit ihr Kostenbewusstsein befördert wird: „Mittelknappheit denke ich, ist im Gegensatz zu früher, allgegenwärtig. Es wird viel gesprochen darüber, schon ab der Assistentenebene, sodass das Bewusstsein für begrenzte Ressourcen inzwischen geschärft ist.“ 
1.4 Tiefeninterviews mit leitenden Ärzten im Rahmen des BMBF Forschungsverbunds „Allokation“"

Tab. 1 Ergebnisse der Tiefeninterviews (Teil 1)

\begin{tabular}{|c|c|c|}
\hline Hauptkategorien & $\begin{array}{l}\text { Subkategorien } \\
\text { 1. Ordnung }\end{array}$ & $\begin{array}{l}\text { Subkategorien } \\
\text { 2. Ordnung }\end{array}$ \\
\hline \multirow{11}{*}{$\begin{array}{l}\text { Status quo: } \\
\text { Mittelknappheit \& } \\
\text { Rationierung }\end{array}$} & \multirow[t]{6}{*}{ Mittelknappheit } & direkte Präsenz \\
\hline & & indirekte Präsenz \\
\hline & & logische Konsequenz \\
\hline & & Gründe \\
\hline & & Kostenbewusstsein \\
\hline & & falsche Darstellung in der Öffentlichkeit \\
\hline & \multirow[t]{5}{*}{ Rationierung } & findet statt \\
\hline & & findet unter bestimmten Bedingungen statt \\
\hline & & $\begin{array}{l}\text { findet unter bestimmten Bedingungen nicht } \\
\text { statt }\end{array}$ \\
\hline & & findet noch nicht statt \\
\hline & & findet unbewusst statt \\
\hline \multirow[t]{17}{*}{ Priorisierungskriterien } & \multirow[t]{6}{*}{ medizinische Kriterien } & Prognose, Nutzen, Risiko als absolute Kriterien \\
\hline & & Prognose, Nutzen, Risiko als relatives Kriterien \\
\hline & & antizipierter Nutzen/Lebensqualität \\
\hline & & Lebenserhaltung \\
\hline & & Schweregrad \\
\hline & & $\begin{array}{l}\text { Probleme der Abgrenzbarkeit oder Cutoff-Be- } \\
\text { stimmung }\end{array}$ \\
\hline & \multirow[t]{4}{*}{ Kosteneffektivität } & relevant \\
\hline & & nicht relevant \\
\hline & & abhängig von der Dateninterpretation \\
\hline & & abhängig von verwendeten Parametern \\
\hline & \multirow[t]{5}{*}{ Lebensalter } & Problem der Definition \\
\hline & & $\begin{array}{l}\text { relatives Kriterium (abhängig von der } \\
\text { Prognose) }\end{array}$ \\
\hline & & $\begin{array}{l}\text { relatives Kriterium (abhängig vom Wunsch des } \\
\text { Patienten) }\end{array}$ \\
\hline & & absolutes Kriterium \\
\hline & & emotionales Kriterium \\
\hline & \multirow[t]{2}{*}{ sonstige Kriterien } & Compliance \\
\hline & & sozialer Beitrag \\
\hline
\end{tabular}


1 Umgang mit begrenzten Mitteln in deutschen Kliniken: empirische Befunde

Tab. 2 Ergebnisse der Tiefeninterviews (Teil 2)

\begin{tabular}{|c|c|c|}
\hline Hauptkategorien & $\begin{array}{l}\text { Subkategorien } \\
\text { 1. Ordnung }\end{array}$ & $\begin{array}{l}\text { Subkategorien } \\
\text { 2. Ordnung }\end{array}$ \\
\hline \multirow{13}{*}{$\begin{array}{l}\text { erschwerende } \\
\text { Bedingungen und } \\
\text { Einflussfaktoren }\end{array}$} & \multirow[t]{6}{*}{ Kontext/System } & finanzielle Grundvoraussetzungen \\
\hline & & Maximal- vs. Regelversorgung \\
\hline & & fehlende (kostengünstigere) Alternativen \\
\hline & & Interaktion zwischen Klinken und Abteilungen \\
\hline & & Schwierigkeiten mit der DRG-Vergütung \\
\hline & & im Budget unberücksichtigte Leistungen \\
\hline & \multirow[t]{5}{*}{ Arzt/Profession } & Konkurrenzdruck \\
\hline & & fehlende Legitimation \\
\hline & & fehlende gesundheitsökonomische Kompetenz \\
\hline & & $\begin{array}{l}\text { unzureichende Vorbereitung auf die Problem- } \\
\text { komplexität }\end{array}$ \\
\hline & & $\begin{array}{l}\text { fehlende Unterstützung bei der Entscheidungs- } \\
\text { findung }\end{array}$ \\
\hline & \multirow[t]{2}{*}{ Patient/Gesellschaft } & Wohl und Wille des Patienten \\
\hline & & $\begin{array}{l}\text { falsche Vorstellungen über Rationierung in der } \\
\text { Gesellschaft }\end{array}$ \\
\hline \multirow[t]{10}{*}{ Konsequenzen } & \multirow[t]{4}{*}{ globale Konsequenzen } & Rollenkonflikte \\
\hline & & Gewissenskonflikte \\
\hline & & neues ärztliches Selbstverständnis \\
\hline & & Unvermeidbarkeit der Rationierung \\
\hline & \multirow{6}{*}{$\begin{array}{l}\text { handlungsbezogene } \\
\text { Konsequenzen }\end{array}$} & Leistungsverdichtung \\
\hline & & Personalabbau \\
\hline & & Überforderung \\
\hline & & emotionaler Stress \\
\hline & & willkürliche Entscheidungen \\
\hline & & Unehrlichkeit \\
\hline
\end{tabular}

Zugleich wurde das fehlende Bewusstsein für die Mittelknappheit in der öffentlichen Diskussion bzw. die falsche Darstellung in der Öffentlichkeit angemahnt: „letztlich ist das eine unehrliche Diskussion. Es wird in der öffentlichen Diskussion immer so dargestellt, als wäre genug Geld vorhanden, wenn man es nur sinnvoll einsetzt.“

Die Frage, ob eine Leistungsbegrenzung in der jeweiligen Klinik stattfindet oder nicht, wurde im Rahmen der Interviews nie dichotom mit "Ja“ oder 
1.4 Tiefeninterviews mit leitenden Ärzten im Rahmen des BMBF Forschungsverbunds „Allokation“

„Nein“ beantwortet. Die Einschätzungen der Interviewpartner diesbezüglich gingen nicht nur auseinander, sondern sie spiegelten den relativen und situativen Gehalt der Begriffe Leistungsbegrenzung oder Rationierung wider. Einige Berichte der Interviewpartner weisen z.B. darauf hin, dass Leistungsbegrenzungen von den jeweiligen Voraussetzungen im Einzelfall abhängen. Diese können unter bestimmten Bedingungen stattfinden: „Wenn trotz Studien die Wirksamkeit, der Effekt von einer teuren Untersuchungs- oder Therapiemethode nicht bekannt ist, bin ich inzwischen so, dass ich die kostengünstigere Variante vorziehen würde, aber nur wenn die Signifikanz fehlt." Oder Leistungsbegrenzungen finden unter bestimmten Bedingungen nicht statt: „Wenn wir sowohl hier auf der [X]-Station wie auch auf der [Y]-Station wirklich von dem Vorteil einer teuren Maßnahme überzeugt sind, dann enthalten wir diese dem Patienten nicht vor."

\section{Priorisierungskriterien}

Zu den von unseren Interviewpartnern genannten Priorisierungskriterien gehören verschiedene medizinische Kriterien, Kosteneffektivität, Alter, Compliance und sozialer Beitrag.

\section{Medizinische Kriterien}

Medizinische Kriterien wie Prognose, Nutzen oder Risiken wurden in einigen Berichten als absolute Kriterien für die Durchführung einer Maßnahme beschrieben: „Also wenn es eine wirklich wissenschaftlich signifikante bedeutsame Besserung ist, gehen wir im Moment so vor, dass das Geld in dem Fall keine Rolle spielt. "In anderen Berichten wiederum wurden sie als relative Kriterien beschrieben, die durchaus gegenüber anderen Kriterien abgewogen werden müssen.

Antizipierende Nutzeneinschätzungen können sich dabei nicht immer auf wissenschaftlich begründete Evidenzen stützen und beruhen dann auf dem erfahrungsgestützten, individuellen ärztlichen Urteil: „Aber auch Lebensqualitätsstudien sind natürlich wichtig, wobei es da in vielen Bereichen nicht viele gibt. Da geht es dann mehr um die Abschätzung, was man selber glaubt, dass sie mehr den Patienten im Ganzen betrachten und überlegen, hilft man ihm jetzt oder schadet man ihm.“

Der Nutzen einer medizinischen Maßnahme als Priorisierungskriterium wurde konkretisiert zum einen durch die Kategorie der Lebenserhaltung: „Also zunächst einmal, Lebenserhaltung ist das größte Kriterium “ und zum anderen durch den Schweregrad der Erkrankung.

Als eine besondere Schwierigkeit bei der Anwendung medizinischer Kriterien wurde die Abgrenzbarkeit oder Cut-off-Bestimmung beschrieben: „gerade dieser Graubereich zwischen eindeutigen Kontraindikationen und eindeutigen In- 
1 Umgang mit begrenzten Mitteln in deutschen Kliniken: empirische Befunde

dikationen ist natürlich das, wobei man immer Schwierigkeiten hat. So eine Entscheidung ist gerade bei der Rationierung schwierig.“

\section{Kosteneffektivität}

Die Kosteneffektivität einer medizinischen Leistung als Priorisierungskriterium wurde unterschiedlich eingeschätzt, zum Teil als relevant: „,Hintergrund: Kosteneffektivitäts-Studien zur kardiovaskulären Therapie, Anm. DS] Also das tue ich persönlich in meinen Alltag schon einbauen natürlich. Das beeinflusst“, aber auch als eher nicht relevant: „Der Kosteneffekt wird ja oft in Untersuchungen so angegeben: Um wie viel besser ist das strukturelle Ergebnis beim Patienten. Sind die weniger abhängig, weniger pflegebedürftig, wenn sie später aus der Klinik entlassen werden? Das sind halt so typische Marker, die aber, so denke ich, für unsere Bilanz, die uns unmittelbar betrifft im Jahr, keine Rolle spielt."

Ob die Kosteneffektivität als Priorisierungskriterium akzeptabel erscheint, wurde von den Ärzten wiederum im Zusammenhang mit bestimmten Bedingungen bewertet. Zum einen ist die praktische Relevanz der Kosteneffektivität abhängig von der Dateninterpretation, zum anderen ist sie abhängig von den verwendeten Parametern: „Die reine Beschränkung auf die Kosten pro gewonnener Lebenszeit, scheint mir pragmatisch aber dann doch nicht weit genug zu greifen.“

\section{Alter}

Auch das Lebensalter wurde in den Interviews als Priorisierungskriterium bei der Begrenzung medizinischer Leistungen genannt. Probleme können sich aber bereits bei der Definition ergeben: „es [die Bedeutung des Alter als Priorisierungskriterium, Anm. DS] ist schwierig zu definieren, weil so vieles da zusammen kommt. Es geht ja nicht nur ums numerische Alter, sondern auch um das biologische Alter der Patienten, um Begleiterkrankungen und das Ausmaß der akuten Erkrankung. “

Das Alter wurde als ein in verschiedener Hinsicht relatives Kriterium beschrieben. Einmal als abhängig von der Prognose: „Ein 85-Jähriger wird seinen Prothesenwechsel eben nie erleben, da muss man auch nicht daran denken, dass der evtl. nach 15 Jahren dann Beschwerden mit der Lockerung oder so Dingen bekommen könnte" und ein anderes mal als abhängig vom Wunsch des Patienten: „Es kommt da wieder drauf an, ob wir auch den Wunsch des Patienten wissen. Wir versuchen natürlich gerade bei älteren Leuten den rauszukriegen. "Das Lebensalter wurde aber auch als absolutes Kriterium benannt: „Man wird sicherlich bei sehr alten Patienten aus den Heimen, dementen Patienten oder ähnlichen überlegen müssen, ob die Maximaltherapie die man einem Jugendlichen oder jungen Patienten angedeihen lässt, dann angebracht ist." 
1.4 Tiefeninterviews mit leitenden Ärzten im Rahmen des BMBF Forschungsverbunds „Allokation“

Darüber hinaus wurde das Alter als ein möglicherweise emotional wirkendes Kriterium beschrieben: „Es gibt sicher bestimmte Situationen in der Klinik, wenn ein Patient einen Infarkt hat und der ist 35, dann rennt man vielleicht ein bisschen schnell und versucht alles 100\%ig zu machen, aber das ist glaube ich ganz subjektiv, das hat auch nichts mit Kosten zu tun, sondern weil das halt einen emotionalen Unterschied ausmacht."

\section{Sonstige Kriterien}

Als weitere, für Priorisierungsentscheidungen relevante Kriterien wurden genannt: die Compliance der Patienten: „Klar, ich würde einem Patienten, von dem ich ausgehe, dass er Aspirin und Clopidogrel nicht regelhaft einsetzen wird, dem würde ich keinen beschichteten Stent implantieren, das ist klar“, und der soziale Beitrag des Patienten: „Einem Patient, der im sozialen Umfeld auch eine Aufgabe hat, der sollte sicherlich auch eine gewisse Bevorzugung genießen.“

\section{Mittelknappheit und ärztliches Handeln: erschwerende Bedingungen und Einflussfaktoren}

Ärztliches Handeln unter Mittelknappheit wird von verschiedenen Faktoren beeinflusst, die sich drei Kategorien (i) Kontext/System, (ii) Arzt/Profession und (iii) Patient/Gesellschaft zuordnen lassen.

\section{Kontext/System}

Als kontext- oder systemabhängig wurden beispielsweise die finanziellen Grundvoraussetzungen an der jeweiligen Klinik beschrieben: „Diese Kliniken haben ja schon eine ganz andere Vorbereitung der Patienten für die Operation, z.B. mit Immunglobulinen vor einer Darmresektion und solchen Sachen, das ist ja bei uns überhaupt kein Thema, da denken wir nicht mal dran. "Praktische Relevanz hat auch die Tatsache der häufig fehlenden kostengünstigeren Alternative.

Einen bedeutenden Einfluss auf den Umgang mit der Mittelknappheit wurde der Interaktion zwischen Kliniken und Abteilungen und den Schwierigkeiten mit der DRG-Vergütung zugeschrieben: „Eine Ablation bei einer ventrikulären Tachykardie kostet mich sehr viel Geld, viele Einmalmaterialien, diese werden mir durch die DRGs nicht entsprechend vergütet. Mach ich dem einen Defibrillator rein, ist dem Patienten auch geholfen, wenn auch anders und nicht kurativ und es wird mir entsprechend bezahlt."

\section{Arzt/Profession}

Von der ärztlichen Profession abhängige Einflussfaktoren im Umgang mit der Mittelknappheit sind der Konkurrenzdruck und die fehlende Legitimation zur Rationierung. Konkurrenzdruck: „Man muss sich ja auch nach außen darstellen, 
wenn ich solche Überlegungen [Kosteneffizienz, Anm. DS] anwende, das würde uns wahrscheinlich mehr schaden als nutzen mittelfristig“, Legitimation: „Mir ist es nicht gestattet, medizinische Leistungen vorzuenthalten. Außer die Solidargemeinschaft sagt, das gibt es nicht.“

Als weitere Einflussfaktoren, die sich auf den einzelnen Arzt beziehen, konnten in unseren Interviews unterschieden werden: die teilweise fehlende gesundheitsökononomische Kompetenz, die unzureichende Vorbereitung auf die Problemkomplexität: „Ich sehe nur eine Reihe von Problemen und eine Reihe von Möglichkeiten, an die man alle durchdenken müsste, die ich aber absolut nicht durchdacht habe“ oder die fehlende Unterstützung bei der Entscheidungsfindung: „Standards gibt es dafür nicht, es gibt keine Leitlinie oder so was, die dem Arzt der etwas nicht tut, hilft."

\section{Patient/Gesellschaft}

Von der Seite der Patienten nehmen Wohl und Wille des Patienten Einfluss auf den Umgang mit dem Kostendruck: „Also die Kosten-Nutzen-Relation muss halt schon stimmen, aber der Patient der darf nicht unberücksichtigt bleiben dabei.“

\section{Mittelknappheit und ärztliches Handeln - Konsequenzen}

Gegenwärtige Konsequenzen der Mittelknappheit, wie sie die befragten Ärzte erleben, konnten 10 verschiedenen Subkategorien zugeordnet werden.

Je nach dem, wie absolut Ärzte ihre Rolle als Anwalt des individuellen Patienten interpretieren, können sich (1.) Rollenkonflikte ergeben: „Ich behandele nicht ganz Deutschland, von daher ist es an sich nicht an mir, die Ressourcen für die restliche Bevölkerung zu rationieren, sondern ich muss meinen Patienten direkt behandeln" oder auch (2.) Gewissenskonflikte. Von einigen Interviewpartnern wurde (3.) ein neues ärztliches Selbstverständnis beschrieben: „Diese Gradwanderung zwischen ökonomischem Handeln einerseits und humanem Denken und Handeln andererseits zu bestehen, ist die Aufgabe meiner Generation denke ich“ oder „Den guten Arzt zeichnet heute nicht nur seine diagnostischen Fähigkeiten aus, sondern auch, aus den gegebenen Möglichkeiten das auszusuchen, was dem Patienten bestens dient, auf der anderen Seite der Solidargemeinschaft das Wenigste kostet.“

Neben diesen globalen Konsequenzen auf das ärztliche Selbstverständnis wurden weitere, eher handlungsbezogene Konsequenzen berichtet, wie (4.) Leistungsverdichtung: „wir müssen mit den gleichen Ressourcen deutlich mehr Leistung erbringen, es kommt zu einer extremen Leistungsverdichtung “, (5.) Personalabbau und weitere damit verbundene Konsequenzen für die Arzt-Patienten-Beziehung: „Personal wird rationiert und unsere Leute können heute mit den Patienten weniger auf der direkten Mensch-zu-Mensch-Ebene arbei- 
1.4 Tiefeninterviews mit leitenden Ärzten im Rahmen des BMBF Forschungsverbunds „Allokation“

ten $[. .$.$] Da wird Zuwendung, Konzentration und damit auch ärztliche und$ menschliche Leistung rationiert“, (6.) Überforderung: „Wir tun das tagtäglich, dass wir da volkswirtschaftlich entscheiden, letztlich auch trotz der Studienlage, aber wie geht man da rational vor?" und (7.) emotionaler Stress.

Als weitere Konsequenzen konnten (8.) willkürliche Entscheidungen ausgemacht werden: „die Implantationszahlen waren stark limitiert und es sollte halt der Erfahrene entscheiden, wer den Stent bekommt und daran haben sich auch alle gehalten. Das war sicherlich problematisch und willkürlich“, (9.) Unehrlichkeit „Ich würde vor dem einzelnen Patienten nicht sagen, ich kann das nicht geben, weil es zu teuer ist.", sowie die letztendliche (10.) Unvermeidbarkeit der Rationierung: „Je häufiger wir uns für eine teure Therapieform entscheiden, weil wir von der Wirksamkeit in dem Fall überzeugt sind, desto häufiger sind wir natürlich dann später verpflichtet, bei Budgetbetrachtungen und anderen Dingen darüber nachzudenken, wo können wir jetzt wieder gegenregulieren um einzusparen."

\subsubsection{Diskussion}

Aus den Ergebnissen der qualitativen Interviews ergeben sich verschiedene Implikationen für die ethische Diskussion zur Kostenbegrenzung im Gesundheitswesen.

\section{Mittelknappheit \& Rationierung}

Zunächst ermöglichen die Aussagen zu Rationierung und Mittelknappheit sowie zu den unterschiedlichen Priorisierungskriterien eine differenziertere und umfassendere Interpretation von der Art und Weise, wie diese Begriffe von Klinikern verstanden und verwendet werden. Die Klärung dieser verschiedenen Begriffsdimensionen kann helfen, die Vermittlungsprobleme in der Diskussion über eine gerechte und effiziente Verteilung von knappen Mitteln im Gesundheitswesen aufzudecken und lösungsorientiert zu bearbeiten. Zum anderen ergeben sich dadurch Konsequenzen für die Interpretation der häufig eindimensional konzipierten quantitativen Studien zur Häufigkeit von Rationierung oder der relativen Wichtigkeit von Priorisierungskriterien wie z.B. des Lebensalters.

Unsere Interviews zeigen auf, dass die Präsenz der Mittelknappheit abhängig vom Arbeitssetting mehr oder weniger direkt wahrgenommen werden kann und dass die Mittelknappheit einmal als logische Konsequenz der Systemstrukturen im deutschen Gesundheitswesen oder ein anderes Mal als begründet durch ärztliches Verhalten verstanden werden kann. Die Bereitschaft, Maßnahmen für eine gerechte Verteilung knapper Ressourcen zu akzeptieren, kann zu nicht unerheblichen Teilen davon abhängig sein, ob und wie die Relevanz oder Präsenz von Mittelknappheit wahrgenommen wird (Mechanic 2002). 
Für die Rationierung trifft dies in ähnlicher Weise zu. Nach dem Erleben einiger Kliniker findet Rationierung statt, nach dem Erleben anderer jedoch nicht. Darüber hinaus gibt es verschiedene andere Einschätzungen, die ein vielschichtigeres Bild der Rationierung ergeben. Demnach findet Rationierung unbewusst, nur unter bestimmten Bedingungen (z.B. bei nicht überzeugender Studienlage) oder eher nicht statt. Offenbar - und dies ist eigentlich wenig überraschend - hängt es von der individuellen Fallkonstellation ab, ob Ärzte medizinische Maßnahmen rationieren oder nicht. Eine wichtige Rolle spielt dabei die verfügbare wissenschaftliche Evidenz für die Wirksamkeit der Maßnahme: Wenn der Nutzen einer Maßnahme klar belegt ist, erhält der Patient diese auch. Wenn aber die Wirksamkeit in klinischen Studien nur unzureichend belegt ist, kommt eher ein Vorenthalten der Maßnahme infrage. Durch diese Differenzierung verschiedener Sichtweisen zum Status quo der Rationierung im deutschen Gesundheitswesen wird deutlich, wie wenig aussagekräftig die Ergebnisse von Fragebogen-Items sind, die lediglich danach fragen, ob rationiert wird oder nicht (Kern et al. 1999). Diese differenziertere Sichtweise auf den Status quo von Rationierung unterstreicht zudem die Notwendigkeit, vermehrt diejenigen Bedingungen zu erforschen, die eine Rationierung befördern bzw. die eine unbewusste Rationierung nach sich ziehen.

\section{Priorisierungskriterien}

Als praxisrelevante Priorisierungskriterien wurden von den interviewten Ärzten medizinische Kriterien, die Kosteneffektivität, das Alter, die Compliance und der sozialer Beitrag thematisiert. Auffällig ist die Tatsache, dass von keinem der Interviewpartner das ethisch kontrovers diskutierte Kriterium der Eigenverantwortung zur Erhaltung der Gesundheit angesprochen wurde (Buyx 2005).

Wie häufig diese Kriterien in der Praxis Anwendung finden und wie verbreitet sie unter praktizierenden Klinikern sind, kann durch die qualitativen Interviews nicht beantwortet werden. Hierfür bedarf es einen quantitativen Studienansatz. In unserer Studie konnte hingegen ähnlich wie bei der Thematik Mittelknappheit und Rationierung eine mehrdimensionale Verwendung der Begriffe einzelner Priorisierungskriterien ermittelt werden. Diese Mehrdimensionalität ist folglich bei der Interpretation von quantitativen Studienergebnissen und ihrer ethischen Analyse zu berücksichtigen. Unter internationalen Studien variiert die Zustimmung zum Priorisierungskriterium des Lebensalters zum Beispiel erheblich. Das Spektrum reicht von 10\% Zustimmung in einer Umfrage unter Allgemeinmedizinern in Wales (Edwards et al. 2003), über 50\% in einer Umfrage unter Kardiologen und Onkologen in den Niederlanden (van Delden et al. 2004) bis zu 88\% unter verschiedenen Ärzten in einer anderen, vor einigen Jahren in den Niederlanden durchgeführten Studie (Tymstra et al. 1993). Gründe für die große Spannbreite der Ergebnisse sind die unterschiedliche Formulierung der Fragestellung, das jeweilige kli- 
1.4 Tiefeninterviews mit leitenden Ärzten im Rahmen des BMBF Forschungsverbunds „Allokation“

nische Arbeitssetting oder das Hintergrundwissen der Antwortende. Zudem hängt die Einschätzung der Relevanz des Lebensalters als Priorisierungskriterium davon ab, wie die befragten Ärzte den Begriff des Lebensalters im Kontext der Ressourcenallokation jeweils verwenden. Die Ergebnisse unserer qualitativen Interviewstudie zeigen, dass insbesondere das Alterskriterium unterschiedlich interpretiert wird. Einige Ärzte verwenden das Lebensalter im Sinne eines wesentlichen Priorisierungskriteriums. Andere Ärzte relativieren das Alterskriterium in Abhängigkeit vom Wunsch des Patienten und von seiner Prognose. Im letzteren Fall dient das Lebensalter als ein - wenngleich auch unscharfer - Surrogatparameter für das eigentliche Priorisierungskriterium der Prognose bzw. des klinischen Nutzens. Die Vielgestaltigkeit und Relativität der Verwendungsweise des Alterskriteriums darf aber nicht darüber hinwegtäuschen, dass dieses Kriterium weiterhin eine Rolle zu spielen scheint, wenn Kliniker über die Allokation knapper Ressourcen reflektieren. Ob das Lebensalter dabei an Überlegungen zum klinischen Nutzen gekoppelt ist oder nicht: Solange dieses Priorisierungskriterium bei konkreten Allokationsentscheidungen nicht explizit benannt wird, besteht eine größere Gefahr für seinen ethisch unangemessenen Einsatz (Strech et al. 2010).

Selbst wenn die Kliniker das Nutzen- oder Prognose-Kriterium als vorrangig in der Ressourcenallokation beschreiben, bleibt auch hier die Schwierigkeit bestehen, einen ausreichenden und intersubjektiv akzeptierten Nutzennachweis zu definieren. Was ist eine „wirklich wissenschaftlich signifikante bedeutsame Besserung“, welche bei Medizinern häufig das stärkste Argument für die Durchführung einer medizinischen Maßnahme zu sein scheint? Was zunächst als objektives und weitgehend anerkanntes Kriterium für eine explizite Rationierung erscheinen mag, liefert in den konkreten klinischen Situationen häufig keine eindeutigen Ergebnisse. Vor dem Hintergrund der oft unsicheren Studienlage zur Nutzen-Schaden- bzw. Kosten-Nutzen-Relation einer medizinischen Maßnahme ist aus ethischer Perspektive ein weiteres Mal ein transparentes und systematisches Vorgehen in der konkreten Entscheidungsfindung zur Ressourcenallokation $\mathrm{zu}$ fordern (Strech $\mathrm{u}$. Marckmann 2010). Wie eine Transparenz zur unzureichenden Studienlage und zu ihren Auswirkungen auf die Entscheidungsfindung in ethisch akzeptabler Weise umgesetzt werden kann, bedarf einer vermehrten Reflexion und Diskussion innerhalb der ethischen und gesundheitsökonomischen Forschung.

\section{Die Praxis der Rationierung: Erschwerende Bedingungen und Konsequenzen}

Neben einer Differenzierung unterschiedlicher Verwendungsweisen von Begriffen wie Rationierung oder Lebensalter als Priorisierungskriterium, liefern die durchgeführten Interviews eine praxisorientierte Übersicht der erschwerenden Bedingungen und Konsequenzen im Hinblick auf den Umgang mit Mittelknappheit in der klinischen Tätigkeit. Dieser Status quo an Rahmenbedingungen gewinnt besondere Bedeutung für die angewandte Ethik, wenn 
versucht werden soll, explizite Instrumente, wie Leitlinien oder Standards, in die Praxis der Kostenkontrolle erfolgreich zu implementieren (Mechanic 2002).

Einige der erschwerenden Bedingungen im Umgang mit der Mittelknappheit zeigen Gründe auf, die für eine Verlagerung der Verteilungsentscheidungen weg von der Arzt-Patienten-Ebene sprechen: die fehlende Unterstützung bei der Entscheidungsfindung zur Kostenreduktion, die besonders schwierige Situation der Ressourcenallokation bei Therapieentscheidungen am Lebensende oder die durch die Mittelknappheit erschwerte Interaktion mit Kollegen und anderen Kliniken.

Die bestehenden erschwerenden Bedingungen zeigen ebenfalls potenzielle Hindernisse bei der Implementierung von expliziten Instrumenten der Kostenbegrenzung auf. Der Konkurrenzdruck unter Ärzten, die Skepsis gegenüber gesundheitsökonomischen Überlegungen aufgrund von fehlender Ausbildung und Kompetenz in diesem Bereich oder die unterschiedlichen finanziellen Grundvoraussetzungen an Krankenhäusern der Maximal- oder Regelversorgung müssen mitbedacht werden um zu verhindern, dass ethisch gut begründete und medizinisch wie ökonomisch sinnvolle Instrumente wie z.B. Kostensensible Leitlinien an den genannten Praxisschwierigkeiten scheitern.

Leistungsbegrenzungen im Gesundheitswesen werden in der Regel von einer gerechtigkeitsethischen Perspektive aus diskutiert (Daniels u. Sabin 2002; Kersting 2002). Die von den Ärzten erlebten Konsequenzen der Mittelknappheit, wie emotionaler Stress, Überforderung oder Gewissenskonflikte, zeigen zudem, welche negativen Auswirkungen bei der Analyse und praktischen Evaluation von Lösungsansätzen im Rahmen der angewandten Ethik mit berücksichtigt werden müssen. Explizite Instrumente zur Leistungsbegrenzung dürfen nicht nur auf die Lösung der gerechtigkeitsethischen Probleme zielen, sondern sollten ebenfalls versuchen, die negativen Auswirkungen auf die Ärzte zu verringern (Hurst et al. 2008).

\subsubsection{Fazit zu den Ergebnisse der Tiefeninterviews}

Die Ergebnisse der Interviewstudie mit Ärzten aus der interventionellen Kardiologie und der Intensivmedizin bieten einen facettenreichen Einblick in die Ressourcenallokation in deutschen Krankenhäusern. Besonders imponiert dabei, wie unterschiedlich die befragten Ärzte die Mittelknappheit wahrnehmen und auf sie reagieren. Ärzte orientieren sich offenbar nicht nur bei klinischen Entscheidungen, sondern auch bei der Allokation knapper Gesundheitsressourcen vor allem an den Besonderheiten des Einzelfalls, wobei hierzu patienten-, maßnahmen- und kontextbezogene Faktoren gehören. Auf der einen Seite ist dies auch aus ethischer Perspektive vorteilhaft, da die Allokationsentscheidungen die medizinische Bedürftigkeit des einzelnen Patienten und den individuell zu erwartenden Nutzen der verfügbaren diagnostischen und therapeutischen Maßnahmen berücksichtigen. Auf der anderen 
1.4 Tiefeninterviews mit leitenden Ärzten im Rahmen des BMBF Forschungsverbunds „Allokation“

Seite besteht aber die Gefahr, dass knappe medizinische Ressourcen nach inkonsistenten und möglicherweise ethisch schlecht begründeten Kriterien verteilt werden. Dies machen auch die Ergebnisse der vorliegenden Interviewstudie deutlich: So spielen offenbar auch nicht patientenbezogene Faktoren wie die finanzielle Gesamtsituation der Klinik, der Konkurrenzdruck oder die erschwerte Kooperation zwischen den Kliniken eine wesentliche Rolle bei knappheitsbedingten Zuteilungsentscheidungen. Auch ethisch eher begründbare Zuteilungskriterien wie der medizinische Nutzen oder die Kosteneffektivität werden von den befragten Ärzten sehr unterschiedlich interpretiert und führen damit möglicherweise zu inkonsistenten Allokationsentscheidungen.

Gleichzeitig bestätigen die Untersuchungen aber auch, dass die Ärzte nicht leichtfertig mit den Allokationsentscheidungen umgehen, im Gegenteil: Die Mittelknappheit und die dadurch hervorgerufenen Verteilungsprobleme können zu Gewissenskonflikten, emotionalem Stress und Gefühlen der Überforderung führen. Dies ist nur allzu verständlich, da Ärzte in der Regel weder ethisch noch gesundheitsökonomisch ausreichend für die Verteilung knapper Ressourcen ausgebildet sind. Darüber hinaus transzendieren gerechte Zuteilungsentscheidungen das traditionelle ärztliche Ethos, das sich allein am Wohlergehen und Willen des einzelnen Patienten orientiert. Dass sich hier aber möglicherweise ein Wandel im ärztlichen Selbstverständnis andeutet, zeigt die Äußerung eines Arztes, dass es zur Aufgabe der heutigen Ärztegeneration gehört, zwischen den Bedürfnissen des einzelnen Patienten und den Anforderungen an eine gerechte Verteilung knapper Ressourcen zu vermitteln. Auch wenn diese Bereitschaft sicher zu begrüßen ist, dürfen Ärzte mit den unvermeidlichen Zuteilungsentscheidungen nicht allein gelassen werden, wie verschiedene Interviewpartner betont haben. Explizite Instrumente der Leistungsbegrenzung wie z.B. Kostensensible Leitlinien (s. Kap. 2) oder auch angepasste Formen der klinischen Ethikberatung (Strech et al. 2010) könnten dabei sicher eine wichtige Rolle spielen.

Darüber hinaus ergeben sich aus den Studienergebnissen methodische Implikationen für empirische Untersuchungen zur Rationierung im Gesundheitswesen. Die für die Verteilung knapper Mittel verwendeten Begriffe wie z.B. der Rationierung oder des Patientenalters als Priorisierungskriterium erweisen sich in der Praxis komplex und multidimensional. Diese Mehrdimensionalität erfordert eine inhaltliche Spezifizierung, sobald die Begriffe in geschlossenen Fragen der quantitativen Interviewforschung (Fragebögen, Surveys) verwendet werden. Ohne eine solche der Frage vorangehende Erläuterung sind die Ergebnisse entsprechender Umfragen nur eingeschränkt verwertbar (Christianson et al. 2005; Strech et al. 2009). In der Ergebnispublikation von Fragebogenstudien sollten diese jeweils verwendeten Hintergrundinformationen zu dem Begriffen genannt werden, um dem Leser eine angemessene Interpretation zu ermöglichen. 
Die hier vorgestellten Ergebnisse geben zwar Aufschluss darüber, welche Spannbreite an Problemen der zunehmende Kostendruck in den Kliniken aufwirft und wie die Ärzte darauf reagieren. Sie erlauben aufgrund des qualitativen Charakters der Interviewstudie aber keine Aussage über die relative Bedeutung der verschiedenen Problemstellungen für ärztliche Entscheidungen. Hierfür ist eine Studie mit quantitativem Design und einer größeren Fallzahl erforderlich. Auch ein Vergleich der Allokationssituation in den beiden Praxisbereichen Kardiologie und Intensivmedizin ist auf der Basis der vorliegenden Interviewergebnisse nicht möglich. In der vorliegenden Studie wurde aber bewusst ein qualitatives Design verwendet, um ein möglichst vielschichtiges und authentisches Bild der Allokationswirklichkeit in den kooperierenden Kliniken zu gewinnen.

\subsection{Postalische Umfragestudie im Rahmen des BMBF Forschungsverbundes „Allokation“}

In 2008 wurde eine repräsentative Umfragestudie im Rahmen des BMBF Forschungsverbundes „Allokation“ durchgeführt. Diese untersuchte die ärztliche Rationierung in Deutschland in den zwei ausgewählten Praxisbereichen, der Kardiologie und der Intensivmedizin. Beide Bereiche stellen besonders interessante Studienobjekte für Rationierungsentscheidungen dar, da sie eine hohe Krankheitslast und viele kostspielige Interventionen mit fraglichem Crenznutzen aufweisen, wodurch sich - so unsere Hypothese - Fragen der Leistungsbegrenzung eher stellen. Sie unterscheiden sich aber in einer für die Forschungsfragen relevanten Hinsicht: Die empirische Nutzenabschätzung ist in der Kardiologie aufgrund der guten Studienlage besser möglich als in der Intensivmedizin, was eine explizite Leistungssteuerung erleichtert. Damit konnten wir verschiedene Einflussfaktoren der Rationierung untersuchen: Wie unterscheidet sich die Häufigkeit von Rationierungen in Abhängigkeit vom Fachbereich, vom Arztstatus (leitende Ärzte vs. nicht leitende Ärzte) und vom Krankenhausträger? Darüber hinaus haben wir untersucht, wie sich die gegenwärtige, vor allem von impliziter Rationierung gekennzeichnete Situation auf die Arbeitszufriedenheit der Ärzte und die Arzt-Patienten-Beziehung auswirkt und wie Ärzte verschiedenen Strategien zum Umgang mit der Mittelknappheit gegenüberstehen.

\subsubsection{Methodik}

Fragebogen: Die Entwicklung des Fragebogens beruht auf (i) den Ergebnissen vorangegangener qualitativer Interviews in der Kardiologie und Intensivmedizin (Huster et al. 2007; Strech et al. 2008; Strech et al. 2009), (ii) den Ergebnissen zweier systematischer Übersichtsarbeit zu internationalen Studien mit ähnlicher Fragestellung (Strech et al. 2008; Strech et al. 2009), sowie (iii) auf 
Panel-Diskussionen im BMBF-Forschungsverbund „Allokation“. Eine erste Version des Fragebogens wurde in kognitiven Interviews mit Klinikärzten unter Anwendung der „thinking aloud“ und „probing“ Technik sowie in einem Pretest evaluiert und modifiziert (Prüfer u. Rexroth 2005; Strech u. Marckmann 2010). Um Interpretationsschwierigkeiten durch unterschiedliche Begriffsverwendungen zu reduzieren, wurde im Fragebogen nicht nach der Häufigkeit von Rationierung, sondern nach der Häufigkeit des Vorenthaltens einer medizinisch nützlichen Leistung aus Kostengründen gefragt. Hierzu wurden fünf- bis sechsstufige Likert-Skalen verwendet. Aus Praktikabilitätsgründen wurden die Studienteilnehmer nicht aufgefordert, ihre Häufigkeitsangaben durch konkrete Beispiele zu erläutern. Sie mussten ebenfalls nicht angeben, mit welcher Evidenz sie die jeweiligen Maßnahmen für welchen Patienten als nützlich angesehen haben. Die Ergebnisse zur Häufigkeit von ärztlicher Rationierung sind deshalb als eine subjektive Einschätzung der Ärzte zu interpretieren. Einschätzungen zu den Auswirkungen von Rationierungen auf Ärzte und Patienten sowie zu Alternativen im Umgang mit Mittelknappheit wurden mit vierstufigen Likert-Skalen erfasst.

Studiendesign \& Sample: In einem ersten Schritt wurde eine bundesweite, disproportional geschichtete Zufallsstichprobe von Kliniken aus dem Krankenhausverzeichnis des Statistischen Bundesamtes gezogen (Statistisches Bundesamt 2007). Schichtungsmerkmale waren a) Kliniken mit internistischen Betten (zur Identifizierung von kardiologischen Abteilungen) und Kliniken mit internistischen und chirurgischen Betten (zur Identifizierung von intensivmedizinischen Abteilungen) und b) Träger des Krankenhauses (öffentlich, freigemeinnützig, privat). Es erfolgten zwei Versendeaktionen unter Berücksichtigung der Rücklaufergebnisse.

Auswertung: Alle statistischen Berechnungen wurden mit SPSS Version 16 durchgeführt. Neben deskriptiven Daten wurde eine binär logistische Regression berechnet, um den Einfluss der Prädiktorvariablen „Art der Abteilung“, „Arztfunktion“ und „Trägerschaft“ auf die als binäres Kriterium erfasste Häufigkeit von Rationierungen (häufig vs. selten/nie) abzuschätzen. Nach Ausschluss nicht vollständig ausgefüllter Fragebögen gingen 490 der 507 Fragebögen in diese Berechnung ein.

\subsubsection{Ergebnisse}

\section{Auswirkungen der Mittelknappheit}

Über zwei Drittel der befragten Ärzte (68\%) bestätigten, dass aufgrund der begrenzt verfügbaren Finanzmittel bei GKV-Versicherten nicht mehr alle medizinisch nützlichen Leistungen erbracht werden könnten. Die überwiegende Mehrheit der Ärzte (82\%) berichtete überdies, der zunehmende Kostendruck beeinträchtige ihre Arbeitszufriedenheit; 78\% gaben an, das Vertrauen der Pa- 
tienten in ihre Ärzte sei durch die Diskussionen über Kostendämpfung und Rationierung gesunken. Nach wie vor sehen sich aber die meisten der befragten Ärzte $(83 \%$ ) verpflichtet, allen Patienten unabhängig von den entstehenden Kosten stets die bestmögliche Diagnostik und Therapie anzubieten.

\section{Absolute und relative Häufigkeit von Rationierungen}

Zur Vermeidung von Interpretationsschwierigkeiten durch unterschiedliche Verwendungen des Begriffs „Rationierung“, fragten wir direkt nach dem zugrunde liegenden Sachverhalt: „Wie häufig haben Sie in den letzten 6 Monaten eine für den Patienten nützliche Maßnahme aus Kostengründen nicht durchgeführt bzw. durch eine preiswertere und zugleich weniger effektive Leistung ersetzt?" Die absoluten als auch relativen Häufigkeiten von Rationierung für die Variablen „Art der Abteilung“, „Arztfunktion“ als auch „Trägerschaft" zeigt Tabelle 3 .

Die Ergebnisse zeigen, dass die Art der Abteilung einen bedeutsamen Vorhersagebeitrag leistet, ob häufig oder selten/nie rationiert wird, während die

Tab. 3 Häufigkeiten der Ausprägungen der Prädiktorvariablen in Abhängigkeit der

Kriteriumsvariablen; Frage: Wie häufig haben Sie in den letzten 6 Monaten eine für den Patienten nützliche Maßnahme aus Kostengründen nicht durchgeführt bzw. durch eine preiswertere und zugleich weniger effektive Leistung ersetzt?

\begin{tabular}{|c|c|c|c|c|}
\hline \multirow[t]{2}{*}{ Prädiktorvariable } & \multicolumn{4}{|c|}{ Kriteriumsvariable } \\
\hline & häufig & selten & nie & N \\
\hline \multicolumn{5}{|l|}{ Art der Abteilung } \\
\hline Kardiologie & 40 (17\%) & $147(62 \%)$ & $51(21 \%)$ & $238(100 \%)$ \\
\hline Intensivmedizin & $26(10 \%)$ & $176(66 \%)$ & $63(24 \%)$ & $265(100 \%)$ \\
\hline Gesamt & $66(13 \%)$ & $323(64 \%)$ & $114(23 \%)$ & $503(100 \%)$ \\
\hline \multicolumn{5}{|l|}{ Arztfunktion } \\
\hline leitend & $22(17 \%)$ & $61(48 \%)$ & $44(35 \%)$ & $127(100 \%)$ \\
\hline nicht-leitend & $43(12 \%)$ & $257(70 \%)$ & $66(18 \%)$ & 366 (100\%) \\
\hline gesamt & 65 (13\%) & $318(65 \%)$ & $110(22 \%)$ & $493(100 \%)$ \\
\hline \multicolumn{5}{|l|}{ Trägerschaft } \\
\hline öffentlich & $25(12 \%)$ & $139(65 \%)$ & $49(23 \%)$ & $213(100 \%)$ \\
\hline freigemeinnützig & $12(10 \%)$ & $86(68 \%)$ & $28(22 \%)$ & $126(100 \%)$ \\
\hline privat & $28(18 \%)$ & $93(60 \%)$ & $34(22 \%)$ & $155(100 \%)$ \\
\hline gesamt & $65(13 \%)$ & 318 (64\%) & $111(23 \%)$ & $494(100 \%)$ \\
\hline
\end{tabular}

Anmerkungen: in Klammern: relative Häufigkeiten gerundet auf volle Prozent; N: Gesamtanzahl (Häufigkeit) der für das Item korrekt ausgefüllten Fragebogen 
Tab. 4 Ergebnisse der binär logistischen Regression

\begin{tabular}{lcccc}
\hline Prädiktorvariablen & $\begin{array}{c}\text { Regressions- } \\
\text { koeffizient B }\end{array}$ & Wald & df & p \\
\hline Art der Abteilung & -.73 & 6,82 & 1 & $.01^{* *}$ \\
\hline Arztfunktion & -.53 & 3,54 & 1 & .07 \\
\hline Trägerschaft & -.30 & 3,54 & 1 & .06 \\
\hline
\end{tabular}

Anmerkungen: Wald: Chi-Quadrat-verteilte Wald-Statistik; df: Freiheitsgrade; p: Irrtumswahrscheinlichkeit; **: statistisch hoch signifikant

Arztfunktion und die Trägerschaft trotz tendenzieller Unterschiede kein statistisch signifikantes Ergebnis erbringen (s. Tab. 4).

\section{Einstellungen der Ärzte zu Ansätzen im Umgang mit Mittelknappheit}

Rationalisierungen fanden eine hohe Zustimmung bei den Studienteilnehmern, auch wenn diese den Patientenpräferenzen entgegenstehen. 90\% der Antwortenden teilten die Auffassung, Ärzte sollten ein ausdrücklich vom Patienten gewünschtes Medikament nicht verschreiben, wenn eine preiswertere und ebenso effektive Alternative verfügbar ist. Die Bereitschaft zur Rationierung ist hingegen deutlich geringer ausgeprägt: Nur 50\% stimmten zu, Ärzte sollten bei Mittelknappheit auf eine preiswertere und geringfügig weniger effektive Alternative ausweichen. Knapp über die Hälfte der Studienteilnehmer (52\%) vertrat die Auffassung, Ärzte sollten, wenn nicht mehr alle medizinisch nützlichen Leistungen finanziert werden können, jeweils im Einzelfall entscheiden, welcher Patient welche Leistungen erhält. Allerdings stimmten $74 \%$ der anschließenden Aussage zu, diese Entscheidungen sollten nach allgemeinen Regeln ,oberhalb “ der individuellen Arzt-Patienten-Beziehung getroffen werden. Die Studienteilnehmer sahen kaum noch Einsparmöglichkeiten im ärztlichen Bereich: Nur ein gutes Drittel (35\%) glaubte, Leistungsbegrenzungen wären überflüssig, wenn die Ärzte selbst wirtschaftlicher arbeiten würden. Die überwiegende Mehrheit der Ärzte (86\%) vertrat hingegen die Auffassung, Leistungsbegrenzungen ließen sich durch ein wirtschaftlicheres Arbeiten der Krankenkassen vermeiden. Deutlich mehr als die Hälfte der Ärzte sieht die Bereitstellung von zusätzlichen Mitteln für den Gesundheitssektor z.B. durch höhere Krankenkassenbeiträge oder Steuern (63\%) oder einen höheren finanziellen Eigenanteil der Patienten (56\%) als einen akzeptablen Weg im Umgang mit der Mittelknappheit.

\subsubsection{Diskussion}

Die repräsentative Befragung von 507 Krankenhausärzten in Kardiologie und Intensivmedizin bestätigt einmal mehr die hohe Prävalenz von Rationierung im deutschen Gesundheitswesen: Über drei Viertel der befragten Ärzte bestä- 
tigten, bereits heute rationieren zu müssen. Einen eher überraschenden Befund ergibt hingegen die erstmals in Deutschland durchgeführte differenzierte Analyse der Häufigkeit von Rationierung: Nur 13\% der Studienteilnehmer gaben an, häufig, d.h. mehr als einmal pro Woche nützliche Maßnahmen aus Kostengründen vorenthalten zu müssen. Bei der Rationierung handelt es sich in deutschen Krankenhäusern zwar um ein weit verbreitetes, aber offenbar (noch) nicht sehr häufiges Phänomen. Wie oft Ärzte rationieren, hängt dabei auch vom Fachbereich ab: Kardiologen rationierten häufiger als Intensivmediziner, was darauf hinweisen könnte, dass Ärzte in lebensbedrohlichen Situationen besonders zurückhaltend mit Rationierungen sind. Ebenfalls im Auge behalten sollte man den Befund, dass der Anteil häufiger Rationierung in Kliniken mit privatem Träger tendenziell etwas größer ist als in öffentlichen oder freigemeinnützigen Kliniken. Ob dies auf einen höheren ökonomischen Druck oder auf ein stärker ausgeprägtes Bewusstsein hinsichtlich eigener Rationierungsentscheidungen zurückzuführen ist, lässt sich aus den Ergebnissen unserer Studie jedoch nicht ableiten.

Obwohl es sich den Ergebnissen zufolge bei der Rationierung noch nicht um ein sehr häufiges Phänomen in deutschen Kliniken handelt, sind die Auswirkungen der Mittelknappheit in der Praxis deutlich spürbar: Über drei Viertel der befragten Ärzte gaben an, der zunehmende Kostendruck beeinträchtige ihre Arbeitszufriedenheit und das Vertrauensverhältnis zwischen Patienten und Ärzten. Eine Erklärung dieses Befundes mag darin liegen, dass sich die überwiegende Mehrheit der Ärzte nach wie vor verpflichtet sieht, ihre Patienten unabhängig von Kostenüberlegungen bestmöglich zu versorgen. Unter den aktuellen Rahmenbedingungen wird es jedoch zunehmend schwieriger diesen moralischen Anspruch zu realisieren, auch wenn es die Studienteilnehmer offenbar immer noch schaffen, vergleichsweise selten den Patienten aus Kostengründen nützliche Maßnahmen vorzuenthalten. Demgegenüber zeigen die Ärzte eine sehr hohe Bereitschaft zur Rationalisierung: Die meisten würden auf eine kostengünstigere, vergleichbar effektive Maßnahme ausweichen, auch gegen die ausdrücklichen Wünsche des Patienten. Allerdings sieht die Mehrheit der befragten Ärzte keine Einsparmöglichkeiten mehr durch eigenes wirtschaftlicheres Handeln, wobei Ergebnisse der Versorgungsforschung insbesondere für die Kardiologie auf Überversorgung und einen unangemessenen Einsatz kostenintensiver Maßnahmen hinweisen (Dissmann u. de Ridder 2002; Fischer u. Avorn 2004). Solange diese Einsparmöglichkeiten von den Ärzten in der Praxis aber nicht gesehen werden, erscheint es problematisch, den Kostendruck in den Kliniken ohne konkrete Unterstützung weiter zu erhöhen. Vor diesem Hintergrund ist es durchaus nachvollziehbar, dass die befragten Ärzte das Einsparpotenzial durch eine wirtschaftlichere Arbeitsweise der Kostenträger deutlich höher einschätzen - obwohl die Krankenkassen derzeit nur eingeschränkte Möglichkeiten haben, die Versorgung zu steuern und damit auch die Effizienz der Leistungserbringung zu verbessern. Die 
Verwaltungskosten der Krankenkassen liegen zudem im internationalen Vergleich auf einem durchschnittlichen Niveau.

Angesichts dieser Befunde könnte man erwarten, dass Ärzte eine Beteiligung an Rationierungsmaßen kategorisch ablehnen. Auch hier ergibt die Untersuchung ein differenzierteres Bild: Immerhin die Hälfte der befragten Ärzte wäre bereit, bei Mittelknappheit auf eine preiswertere und geringfügig weniger effektive Alternative auszuweichen, was nach der hier zugrunde liegenden Definition eine Rationierung darstellt. Ebenfalls knapp über die Hälfte der Studienteilnehmer vertrat die Auffassung, Ärzte sollten jeweils im Einzelfall Verantwortung für Rationierungen übernehmen und entscheiden, welcher Patient welche Leistungen erhält, wenn nicht mehr alle nützlichen Leistungen finanziert werden können. Dass gleichzeitig drei Viertel der Befragten einer oberhalb der individuellen Arzt-Patient-Beziehung geregelten Rationierung zustimmten, unterstreicht die ambivalente Einstellung gegenüber ärztlicher Rationierung. Verständlicherweise möchten sich die befragten Ärzte einen möglichst großen Entscheidungsspielraum erhalten. Dies ist aber nur möglich, wenn sie selbst Verantwortung für die Zuteilung knapper Ressourcen übernehmen und Rationierungsentscheidungen „am Krankenbett“ treffen. Dann müssten Ärzte aber eine Aufgabe übernehmen, für die sie keine spezifische Ausbildung besitzen und die sie in Konflikt mit ihrer traditionellen Rolle als Anwalt ihres Patienten bringen kann. Insofern ist es ebenfalls nachvollziehbar, wenn die Mehrheit der befragten Ärzte eine Regelung „oberhalb“ der individuellen Arzt-Patient-Beziehung bevorzugt.

\subsubsection{Kommentar zur Rezeption dieser Studienergebnisse}

Insbesondere das Ergebnis, dass $78 \%$ der Ärzte mindestens einmal innerhalb der letzten 6 Monate und 13\% der Ärzte sogar mindestens einmal pro Woche potenziell nützliche Maßnahmen allein aus Kostenüberlegungen vorenthalten haben (was einer Rationierung entspricht), wurde in öffentlichen Medien wie auch in Fachkreisen intensiv und kontrovers diskutiert (Sonnet 2.5.2010; MDR [Fakt ist ...!] 5.10.2009; SWR2 Forum 18.3.2010; Albrecht 2009). Ein Jahr nach der Publikation der oben beschriebenen Ergebnisse haben wir in einem ergänzenden Beitrag dafür argumentiert, dass die Zahlen eine höhere interne und externe Validität aufweisen, als von Kritikern häufig eingewendet wird. Folgende vier Einwände wurden entkräftet bzw. in ihrer praktischen Bedeutung relativiert (Strech u. Marckmann 2010): 1) Die teilnehmenden Ärzte haben die Frage falsch verstanden, 2) Gemessen wurde lediglich ein politisches Statement der Ärzteschaft, 3) Gemessen wurde nur eine „weiche“ Rationierung, 4) Gemessen wurde nur eine „gefühlte“ Rationierung.

Dort wurden zudem weitere Studienergebnisse berichtet, die aufzeigen, dass neben diagnostischen und pflegerischen Maßnahmen auch konkrete therapeutische Maßnahmen (z.B. das Reserveantibiotikum Linezolid oder auch 
1 Umgang mit begrenzten Mitteln in deutschen Kliniken: empirische Befunde

Tab. 5 Frage: „Wie häufig haben Sie oder einer Ihrer Kollegen in Ihrer Abteilung in den letzten 6 Monaten aus Kostengründen eine der folgenden Maßnahmen aufgeschoben oder nicht durchgeführt, obwohl sie nach Ihrer Auffassung für den Patienten nützlich gewesen wären?"

\begin{tabular}{|c|c|c|c|c|}
\hline Interventionstypen & $\begin{array}{l}\text { mindestens } \\
\text { einmal pro } \\
\text { Woche }\end{array}$ & $\begin{array}{l}\text { mindestens } \\
\text { einmal im } \\
\text { Monat }\end{array}$ & $\begin{array}{c}\text { seltener als einmal im } \\
\text { Monat }\end{array}$ & nie \\
\hline $\begin{array}{l}\text { Anordnung pflegerischer } \\
\text { Maßnahmen }\end{array}$ & $13 \%$ & $13 \%$ & $27 \%$ & $46 \%$ \\
\hline diagnostische Maßnahmen & $10 \%$ & $31 \%$ & $28 \%$ & $31 \%$ \\
\hline $\begin{array}{l}\text { nichtmedikamentöse } \\
\text { therapeutische Maßnahmen }\end{array}$ & $7 \%$ & $15 \%$ & $37 \%$ & $41 \%$ \\
\hline medikamentöse Maßnahmen & $5 \%$ & $17 \%$ & $36 \%$ & $42 \%$ \\
\hline
\end{tabular}

Tab. 6 Frage entsprechend Tabelle 5

\begin{tabular}{|lccccc}
\hline & $\begin{array}{c}\text { mindestens } \\
\text { einmal } \\
\text { pro Woche }\end{array}$ & $\begin{array}{c}\text { mindestens } \\
\text { einmal } \\
\text { im Monat }\end{array}$ & $\begin{array}{c}\text { seltener als } \\
\text { einmal } \\
\text { im Monat }\end{array}$ & $\begin{array}{c}\text { nicht verfügbar } \\
\text { in meiner } \\
\text { Abteilung }\end{array}$ \\
\hline $\begin{array}{l}\text { Fresh Frozen Plasma (FFP) } \\
\begin{array}{l}\text { NovoSeven@ (rekombinanter } \\
\text { Gerinnnungsfaltor VIla) }\end{array}\end{array}$ & $2 \%$ & $3 \%$ & $8 \%$ & $87 \%$ & $0 \%$ \\
\hline Xigris@ (Drotrecogin alfa) & $1 \%$ & $2 \%$ & $12 \%$ & $85 \%$ & $18 \%$ \\
\hline Zyvoxid@ (Linezolid) & $1 \%$ & $2 \%$ & $18 \%$ & $79 \%$ & $29 \%$ \\
\hline
\end{tabular}

Tab. 7 Frage entsprechend Tabelle 5

\begin{tabular}{lccccc} 
& $\begin{array}{c}\text { mindestens } \\
\text { einmal pro } \\
\text { Woche }\end{array}$ & $\begin{array}{c}\text { mindestens } \\
\text { einmal im } \\
\text { Monat }\end{array}$ & $\begin{array}{c}\text { seltener als } \\
\text { einmal im } \\
\text { Monat }\end{array}$ & $\begin{array}{r}\text { nicht verfügbar } \\
\text { in meiner } \\
\text { Abteilung }\end{array}$ \\
\hline $\begin{array}{l}\text { Interventionelle Kardiologie } \\
\text { Kardiale Resynchronisation } \\
\text { (CRT) }\end{array}$ & $1 \%$ & $2 \%$ & $24 \%$ & $73 \%$ & $24 \%$ \\
\hline $\begin{array}{l}\text { Implantierbare Defibrillatoren } \\
\text { (ICD) }\end{array}$ & $0 \%$ & $3 \%$ & $28 \%$ & $69 \%$ & $20 \%$ \\
\hline $\begin{array}{l}\text { Linksherzkatheter mit } \\
\text { Koronarangiographie }\end{array}$ & $0 \%$ & $6 \%$ & $26 \%$ & $68 \%$ & $11 \%$ \\
\hline $\begin{array}{l}\text { Medikamente-freisetzende } \\
\text { Stents (DES) }\end{array}$ & $4 \%$ & $6 \%$ & $27 \%$ & $63 \%$ & $16 \%$ \\
\hline
\end{tabular}

implantierbare Defibrillatoren) rationiert werden (s. Tab. 5, 6 u. 7), wenngleich, logischer Weise, in weniger hohen Prozentzahlen als für die Maßnahmen übergreifende Fragestellung. 


\subsection{Diskussion}

An anderer Stelle wurden explizit und ausführlich diejenigen Einwände kommentiert, welche darauf abzielen, die Bedeutung der in Abschnitt 5 dargestellten Studienergebnisse in ihrer Validität zu relativieren (Strech u. Marckmann 2010). Dies soll an dieser Stelle nicht wiederholt werden. Als Konsequenz aus der Validitätskritik wird oft geschlussfolgert, dass kein unmittelbarer Handlungsbedarf im Umgang mit Rationierungsproblemen in der ärztlichen Praxis bestehe. Tatsächlich wäre es möglich, in einem noch aufwendigeren Studiendesign den Status quo gegenwärtiger Rationierung zu erfassen (z.B. in ethnografischen Studien oder in der Auswertung von Krankenakten mit anschließendem Interview des jeweils zuständigen Arztes). Diese Studien wären mit einem erheblichen methodischen Aufwand verbunden (Strech u. Marckmann 2010). Hier stellt sich die Frage, ob wir den möglichen Zugewinn an Validität tatsächlich benötigen, um eine Beschäftigung mit dem Problem der Rationierung in deutschen Kliniken und der Priorisierung auf Gesundheitssystemebene hinreichend zu legitimieren. Aus den an anderer Stelle dargelegten Gründen (Strech u. Marckmann 2010) können wir davon ausgehen, dass die in Kapitel 5 dargestellten Ergebnisse aufgrund von Missverständnissen oder politischer Motivation der befragten Ärzte zumindest nicht in erheblichem Ausmaß verzerrt sind. Insgesamt haben wir damit einen relativ verlässlichen, wenn auch nicht absolut sicheren empirischen Nachweis, dass in deutschen Kliniken subjektiv gefühlte wie auch objektiv zu bestätigende Rationierung stattfindet. Ob die Evidenz aus den verschiedenen qualitativen und quantitativen Studien hinreichend verlässlich ist, um entsprechende Konsequenzen daraus zu ziehen, lässt sich nur im Hinblick auf die konkreten Handlungsoptionen selbst bestimmen. Im Folgenden werden deshalb einige mögliche Konsequenzen aufgezeigt und dabei jeweils geprüft, ob ein noch validerer empirischer Nachweis als Rechtfertigung erforderlich wäre.

Aus den genannten Studienergebnissen lassen sich - in Verbindung mit ethischen Aspekten - verschiedene Handlungsaufforderungen für die Politik, die gemeinsame Selbstverwaltung und andere Akteure im deutschen Gesundheitswesen wie Patientenvertreter und medizinische Fachgesellschaften ableiten. Ein ethisch zentraler Aspekt betrifft das Potenzial struktureller Benachteiligungen vulnerabler Patientengruppen durch die gegenwärtig vorherrschende implizite (verdeckte) Form von Rationierung (Strech et al. 2008; Strech et al. 2008). Diese ist zum Beispiel für ältere Menschen auch empirisch belegt (Brockmann 2002).

Dass es sich bei den ableitbaren Handlungsoptionen nicht zwangsläufig um mehr Geld für das Gesundheitssystem handeln muss bzw. dass mit dieser das Problem eher verdeckenden Option auch gesundheitsbezogene Opportunitätskosten verbunden sein können, ist an verschiedenen Stellen plausibel dargestellt worden (Marckmann 2007; Huster 2008). Höhere Gesundheitsausgaben können 
nur mit Einschränkungen in anderen sozialstaatlichen Bereichen erkauft werden, was wiederum negative Auswirkungen auf den Gesundheitszustand der Menschen und auf die gerechte Verteilung von Gesundheit haben könnte.

Vielmehr sollte eine konzertierte Aktion der oben genannten Akteure für eine verbesserte Qualität der entscheidungsrelevanten Evidenz zu Nutzen, Schaden und Kosten medizinischer Maßnahmen höchste Priorität genießen (Lelgemann u. Sauerland 2010; Schott et al. 2010; Strech 2010 ). Zum einen können dadurch - im Sinne einer Rationalisierung - Ressourcen freigesetzt werden, was den mit unserer Studie empirisch belegten Druck auf die Ärzte reduzieren könnte, auf potenziell nützliche Maßnahmen zu verzichten. Zum anderen würde damit die Grundlage für einen medizinisch rationalen und ethisch vertretbaren Einsatz knapper Ressourcen geschaffen.

Die ebenfalls sinnvolle und notwendige Beschäftigung mit Priorisierung und Rationierung darf davon nicht ablenken. Sie sollte aber auch nicht in Abrede gestellt werden (ZEKO 2007). Häufig wird argumentiert, es sei ethisch nicht vertretbar, über Rationierung nachzudenken, solange es noch Wirtschaftlichkeitsreserven im System gäbe. Dieses Argument mag auf der konzeptionellen Ebene durchaus zutreffen, geht aber an der Realität des Gesundheitswesens vorbei: Man wird nie ein vollkommen effizientes System erreichen. Auch wenn noch Über- und Fehlversorgung vorhanden sind, sollte man bei bestehender Mittelknappheit die Beschäftigung mit einer ebenfalls existierenden Rationierung nicht ausblenden. Dies gilt insbesondere dann, wenn durch die implizite Form der Rationierung vulnerable Patientengruppen benachteiligt werden.

Problematisch ist es allerdings, wenn mit der zunehmenden Diskussion zur Notwendigkeit von Priorisierungen und Rationierungen nicht zugleich eine deutlich intensivierte Anstrengung zum Abbau von Über-, Unter- und Fehlversorgung einhergeht. Auch eine in ihren Prozessen ethisch akzeptable Rationierung bleibt ethisch problematisch, wenn parallel nicht intensiv an der Ausschöpfung weiterer Rationalisierungsreserven gearbeitet wird. Aufgrund der Schärfe der Problematik, erscheint die alleinige Selbstverpflichtung zu vermehrter Rationalisierung in der Ärzteschaft zunächst nicht ausreichend (Fuchs 2010). Verhältnismäßiger wäre eine Stellungnahme der Ärzteschaft, welche z.B. konkrete Vorhaben benennt, mit denen Rationalisierungsreserven parallel zu den Rationierungsdiskursen weiter ausgeschöpft werden. Um die Ernsthaftigkeit zu bezeugen, mit der man das Primat der Rationalisierung vor Rationierung bekundet, wäre es u.a. angebracht, konkrete Ziele der Vorhaben bzw. „Benchmarks für eine angemessene Effizienz“ zu benennen (Strech $u$. Danis 2014).

Vor dem Hintergrund der Ergebnisse aus der Versorgungsforschung, welche international wie auch spezifisch für Deutschland eine weiterhin bestehende Unter-, Über- und Fehlversorgung anzeigen (Sachverständigenrat für die Kon- 
zertierte Aktion im Gesundheitswesen 2001; Dissmann u. de Ridder 2002; Bach 2009), ist es durchaus nachvollziehbar, dass von Seite der gesetzlichen Krankenkassen wie von der Bundespolitik befürchtet wird, dass die Ärzteschaft die Themen Priorisierung und Rationierung zumindest mitbenutzt, um Mittelerhöhungen für ihren Aufgabenbereich durchzusetzen (Storz u. Egger 2010). Qualitätsdefizite in der ärztlichen Versorgung würden dadurch konserviert.

Insgesamt liefern die verfügbaren empirischen Studien einen hinreichend validen Nachweis über Rationierung im deutschen Gesundheitswesen, um entsprechende Maßnahmen zur Verbesserung des Umgangs mit knappen Gesundheitsressourcen zu rechtfertigen. Weitere, noch verlässlichere Studien wären zwar theoretisch machbar, der zusätzliche Erkenntnisgewinn würde aber kaum den erheblichen methodischen Aufwand rechtfertigen. Stattdessen sollten alle Akteure im Gesundheitswesen - Politik, Kostenträger, Leistungserbringer, Patientenvertreter - ihre Bemühungen um einen medizinisch rationalen, ethisch wie rechtlich vertretbaren und ökonomisch sinnvollen Umgang mit den begrenzt verfügbaren Gesundheitsressourcen intensivieren. Eine optimierte Nutzenbewertung kann dabei nicht nur einen Beitrag zur Rationalisierung und Verbesserung der Versorgungsqualität leisten, sondern schafft darüber hinaus eine wesentliche Voraussetzung für eine ethisch vertretbare, evidenzbasierte Priorisierung und Rationierung.

\section{Literatur}

Albrecht, H. (2009). Medizin am Limit. Welche Behandlung sollen wir uns noch leisten? Wir weigern uns, über diese zentrale Frage zu diskutieren. Die ZEIT 51. URL: http://www.zeit.de/2009/51/M-Priorisierung (Zugriff am 21.04.2015).

Ayres, P.J. (1996). Rationing health care: Views from general practice. Social Science \& Medicine 42: 1021-1025.

Bach, P.B. (2009). Limits on Medicare's ability to control rising spending on cancer drugs. N Engl J Med 360(6): 626-633.

Baines, D.L., K.H. Tolley and D.K. Whynes (1998). The ethics of resource allocation: the views of general practitioners in Lincolnshire, U.K. Soc Sci Med 47(10): 1555-1564.

BÄK (2008). Ulmer Papier. Gesundheitspolitische Leitsätze der Ärzteschaft. Beschluss des 111. Deutscher Ärztetag 2008. Bundes. Bundesärztekammer (BÄK). Berlin.

Bernat, J.L., S.P. Ringel, B.G. Vickrey, et al. (1997). Attitudes of US neurologists concerning the ethical dimensions of managed care. Neurology 49(1): 4-13.

Beske, F. (2010). Bedarfsgerechte Gesundheitsversorgung bei begrenzten Mitteln. Situationsanalyse, internationaler Vergleich, Handlungsoptionen. Kiel.

Boldt, J. and T. Schöllhorn (2008). Rationierung ist längst Realität. Ergebnisse einer Fragebogenaktion auf deutschen Intensivstationen. Deutsches Ärzteblatt 105(19): A995-997.

Brockmann, H. (2002). Why is less money spent on health care for the elderly than for the rest of the population? Health care rationing in German hospitals. Soc Sci Med 55(4): 593-608.

Buhr, P. and S. Klinke (2006). Qualitative Folgen der DRG-Einführung für Arbeitsbedingungen und Versorgung im Krankenhaus unter Bedingungen fortgesetzter Budgetierung. Berlin, Wissenschaftszentrum Berlin für Sozialforschung (WZB).

Buyx, A. (2005). Eigenverantwortung als Verteilungskriterium im Gesundheitswesen - Theoretische Grundlagen und praktische Umsetzung. Ethik in der Medizin 17(4): 269-283. 
Carlsen, B. and 0.F. Norheim (2005). Saying no is no easy matter a qualitative study of competing concerns in rationing decisions in general practice. BMC Health Serv Res 5: 70.

Christianson, I.B., L.H. Warrick and D.R. Wholey (2005). Physicians' Perceptions of Manged Care: A Review of the Literature. Medical Care Research and Review 72(6): 635-675.

Daniels, N. and J.E. Sabin (2002). Setting limits fairly. Oxford, Oxford University Press.

Dissmann, W. and M. de Ridder (2002). The soft science of German cardiology. Lancet 359(9322): 2027-2029.

Edwards, R.T., A. Boland, C. Wilkinson, et al. (2003). Clinical and lay preferences for the explicit prioritisation of elective waiting lists: survey evidence from Wales. Health Policy 63(3): 229-237.

Fischer, M.A. and I. Avorn (2004). Economic implications of evidence-based prescribing for hypertension: can better care cost less? JAMA 291(15): 1850-1856.

Fojo, T. and C. Grady (2009). How much is life worth: cetuximab, non-small cell lung cancer, and the $\$ 440$ billion question. I Natl Cancer Inst 101(15): 1044-1048.

Fuchs, C. (2010). Demografischer Wandel und Notwendigkeit der Priorisierung im Gesundheitswesen: Positionsbestimmung der Arzteschaft. Bundesgesundheitsblatt Gesundheitsforschung Gesundheitsschutz 53(5): 435-440.

Hurst, S.A., S.C. Hull, G. DuVal, et al. (2005). Physicians' responses to resource constraints. Arch Intern Med 165(6): 639-644.

Hurst, S.A., S. Reiter-Theil, A.M. Slowther, et al. (2008). Should ethics consultants help clinicians face scarcity in their practice? I Med Ethics 34(4): 241-246.

Hurst, S.A., A.M. Slowther, R. Forde, et al. (2006). Prevalence and Determinants of Physician Bedside Rationing: Data from Europe. I Gen Intern Med 21(11): 1138-1143.

Huster, S. (2008). „Hier finden wir zwar nichts, aber wir sehen wenigstens etwas“. Zum Verhältnis von Gesundheitsversorgung und Public Health. Bochum, Zentrum für Medizinische Ethik.

Huster, S., D. Strech, G. Marckmann, et al. (2007). Implizite Rationierung als Rechtsproblem. Ergebnisse einer qualitativen Interviewstudie zur Situation in deutschen Krankenhäusern Medizinrecht 25(12): 703-706.

Jones, I.R., L. Berney, M. Kelly, et al. (2004). Is patient involvement possible when decisions involve scarce resources? A qualitative study of decision-making in primary care. Soc Sci Med 59(1): 93-102.

Katzenmeier, C. (2010). Engpasse medizinischer Versorgung-Rechtliche Implikationen. Z Evid Fortbild Qual Gesundhwes 104(5): 364-370.

Kent, D. (2010). Just-as-good Medicine. Less expensive, lower-quality innovations abound in every economic sector-except medicine. American Scientist 98(2): 98-102.

Kern, A.0., F. Beske and H. Lescow (1999). Leistungseinschränkung oder Rationierung im Gesundheitswesen? Deutsches Ärzteblatt 96(3): A113-A117.

Kersting, W. (2002). Gerechtigkeitsethische Überlegungen zur Gesundheitsversorgung. Gesundheitsökonomische Evaluationen. 0. Schöffski and I.-M. von der Schulenburg. Berlin, Springer-Verlag: 25-49.

Kuhlmann, E. (1999). Im Spannungsfeld zwischen Informed Consent und konfliktvermeidender Fehlinformation: Patientenaufklärung unter ökonomischen Zwängen. Ergebnisse einer empirischen Studie. Ethik in der Medizin 11: 146-161.

Lelgemann, M. and S. Sauerland (2010). Gefälschte Studien und nicht publizierte Daten: Auswirkung auf die Erarbeitung von Leitlinien und evidenzbasierten Empfehlungen [im Druck]. Zeitschrift für Evidenz, Fortbildung und Qualität im Gesundheitswesen (ZEFQ)(4).

Marckmann, G. (2007). Zwischen Skylla und Charybdis: Reformoptionen im Gesundheitswesen aus ethischer Perspektive. Gesundheitsökonomie und Qualitätsmanagement 12: 96-100.

MDR (Fakt ist ...!) (5.10.2009). „Risiko mit Nebenwirkungen. Altersgrenzen für teure Medizin?“ Fakt ist ...!

Mechanic, D. (2002)."Socio-cultural implications of changing organizational technologies in the provision of care." Social Science \& Medicine 54: 459-467.

Pearlman, R.A. and H.E. Starks (2001). Quantitative Surveys. Methods in Medical Ethics. I. Sugarman and D.P. Sulmasy. Washington D.C., Georgetown University Press: 192-206.

Prüfer, P. and M. Rexroth (2005). Kognitive Interviews. Mannheim, ZUMA.

Raspe, H. and I. Schulze (2013). Medizinische Versorgung: Ärztlich unterstützte Priorisierung ist notwendig und hilfreich. Deutsches Ärzteblatt 110(22): A1091-1096. 
Reeleder, D., D.K. Martin, C. Keresztes, et al. (2005). What do hospital decision-makers in Ontario, Canada, have to say about the fairness of priority setting in their institutions? BMC Health Serv Res 5(1): 8.

Reichert, S., T. Simon and E.A. Halm (2000). Physicians' attitudes about prescribing and knowledge of the costs of common medications. Arch Intern Med 160(18): 2799-2803.

Sachverständigenrat für die Konzertierte Aktion im Gesundheitswesen (2001). Bedarfsgerechtigkeit und Wirtschaftlichkeit. Gutachten 2000/2001, Kurzfassung. Bonn.

Schott, G., H. Pachl, U. Limbach, et al. (2010). Finanzierung von Arzneimittelstudien durch pharmazeutische Unternehmen und die Folgen - Teil 1: Qualitative systematische Literaturübersicht zum Einfluss auf Studienergebnisse, -protokoll und -qualität. Dtsch Arztebl 107(16): 279-285.

Schultheiss, C. (2004). Im Räderwerk impliziter Rationierung. Auswirkungen der Kostendämpfung im deutschen Gesundheitswesen. Teil I: Rationierung in verschiedenen Leistungsbereichen. psychoneuro 30: 221-226.

Sonnet, C. (2.5.2010). Medizinische Versorgung. Jeder kriegt, was er braucht. Von wegen! FAZ. URL: http:// www.faz.net/aktuell/wirtschaft/wirtschaftspolitik/medizinische-versorgung-jeder-kriegt-was-er-brauchtvon-wegen-11281.html (Zugriff am 21.04.2015).

Statistisches Bundesamt (2007). Krankenhausverzeichnis. Statistische Ämter des Bundes und der Länder. Stand 31.12.2005. Wiesbaden.

Storz, P. and B. Egger (2010). Die Debatte über Priorisierung und Rationierung in der Gesetzlichen Krankenversicherung: überfällig oder überflüssig? Gesundheits- und Sozialpolitik(1): 11-18.

Strauss, A.L. and J.M. Corbin (1996). Grounded Theory: Grundlagen Qualitativer Sozialforschung. Weinheim, Beltz PVU.

Strech, D. (2010). Zur Ethik einer restriktiven Regulierung der Studienregistrierung (online first). Ethik in der Medizin.

Strech, D., K. Börchers, D. Freyer, et al. (2008). Ärztliches Handeln bei Mittelknappheit. Ergebnisse einer qualitativen Interviewstudie. Ethik in der Medizin 20(2): 94-109.

Strech, D. and M. Danis (2014). How can bedside rationing be justified despite coexisting inefficiency? The need for 'benchmarks of efficiency'. I Med Ethics 40(2): 89-93.

Strech, D., D. Freyer, K. Börchers, et al. (2009). Herausforderungen expliziter Leistungsbegrenzungen durch kostensensible Leitlinien. Ergebnisse einer qualitativen Interviewstudie mit leitenden Klinikärzten. Gesundheitsökonomie und Qualitätsmanagement 14: 38-43.

Strech, D., S. Hurst and M. Danis (2010). The Role of Ethics Committees and Ethics Consultation in Allocation Decisions: A 4-Stage Process. Med Care 48(9): 821-826.

Strech, D. and G. Marckmann (2010). Wird in deutschen Kliniken rationiert oder nicht? Wie genau wir es wissen und warum es nicht die wichtigste Frage sein sollte. Dtsch Med Wochenschr 135(30): 1498-1502.

Strech, D., G. Persad, G. Marckmann, et al. (2009). Are physicians willing to ration health care? Conflicting findings in a systematic review of survey research. Health Policy 90(2-3): 113-124.

Strech, D., M. Synofzik and G. Marckmann (2008). How physicians allocate scarce resources at the bedside: a systematic review of qualitative studies. I Med Philos 33(1): 80-99.

Stronks, K., A.M. Strijbis, J.F. Wendte, et al. (1997). Who should decide? Qualitative analysis of panel data from public, patients, healthcare professionals, and insurers on priorities in health care. BMI 315(7100): 92-96.

SWR2 Forum (18.3.2010). Medizin am Limit - Welche Behandlungen bleiben bezahlbar? URL: http://podster. de/episode/1314203 (Zugriff am 21.04.2015).

Truog, R.D. (2009). Screening mammography and the "r" word. N Engl / Med 361(26): 2501-2503.

Tymstra, T., M. Andela, T. Tymstra, et al. (1993). Opinions of Dutch physicians, nurses, and citizens on health care policy, rationing, and technology. JAMA 270(24): 2995-2999.

van Delden, I.J., A.M. Vrakking, A. van der Heide, et al. (2004). Medical decision making in scarcity situations. I Med Ethics 30(2): 207-211.

Ward, N.S., J.M. Teno, J.R. Curtis, et al. (2008). Perceptions of cost constraints, resource limitations, and rationing in United States intensive care units: results of a national survey. Crit Care Med 36(2): 471-476.

ZEKO (2007). Priorisierung medizinischer Leistungen im System der Gesetzlichen Krankenversicherung (GKV). Zentrale Kommission zur Wahrung ethischer Grundsätze in der Medizin und ihren Grenzgebieten (Zentrale Ethikkommission) bei der Bundesärztekammer. Deutsches Ärzteblatt 104(40): A2750-2754. 